\title{
Über eine burgundische Gesandtschaft an den kaiserlichen und päpstlichen Hof im Jahre 1460. Ton Otto Cartellieri.
}

Mit Studien über die Geschichte Herzog Philipps des Guten ron Burgund beschäftigt, fand ich in Brüssel ${ }^{1)}$ die Abschrift, dann in Lilie ${ }^{2}$ ) das Original einer bemerkenswerten Gesandtschaftsinstruktion, die einmal Aufschlüsse gibt über den berühmten, in Wort und Bild gefeierten Türken-Kongress von Nantua, die dann aber auch einen interessanten. bislang fast ganz unbeachteten ${ }^{3}$ ) Beitrag liefert zu den langwierigen Bündnis- und Heirats-Verhandlungen zwischen den Häusern Habsburg und Valois-Burgund.

1) Bibl. roy. 7246 in Sammelbande 7243-7251, a as welchem einzelne Teile herangezogen wurden ron G. Du Fresne de Beaucourt, Hist. de Charles TII Bd. VI (Paris 1891) $211 \mathrm{ff}$. 27.2 ff. Sowohl in Brüssel als in Lille wurde ich sehr liebenswürdig aufgenommen; ich benutze sehr gern die Gelegenheit, meinen rerbindlichsten Dank allen zu sagen, die meine Arbeiten förderten: in Brüssel den Herren Henry Hymans; J. van den Ghern; A. Bayot; J. Cuvelier; F. Laloire: H. Nelis; G. des Marez. In Lille: Den Herren J. Finot; J. Vermaere; C. Delattre. Für gütige Auskunft bin ich Herrn Hofrat Professor Dr. A. Bachmann rerbunden.

2) Arch. du Nord, Chambre des Comptes de Lille, Art. B nouveau 855 ('Trésor des Chartes nr. 15 992).

8) J. Chmel, Gesch. Faiser Friedrichs IV. und seines Sobnes Maximilians II (Hamburg 1843) $492 \mathrm{Anm}$. 1, der aut die Verhandlungen 2 wischen Friedrich und Philipp in den vierziger Jahren nüher eingeht, spricht sein Bedauern aus, keine weiteren Spuren von Unterhandlungen aufgefunden zu haben, die sicherlich nicht ex abrupto aufgehört hätten. Gottlieb Krause, Beziehungen zwischen Habsburg und Burgund bis zum Ausgang der Trieres Zusammenkunft im Jabre 1473 (Gött. Diss. 1876) und A. Leroux, Nouv. recherches critiques sur les relations polit. de la 
Die Instruktion ist zu Brüssel am 1. Mai 1460 ausgestellt für den Magister Anton Hanneron, einen bewährten Diener des burgundischen Hauses ${ }^{1}$, der wie schon kurz vorher an den kaiserlichen und päpstlichen Hof geschickt wird. Im Sommer 1459 Mitglied von Philipps des Guten Gesandtschaft nilch Mantua ${ }^{2}$ ), erhält Hauneron jetzt deu Auftrag. den Kaiser über die dort getroffenel Abmachungen seinen Bericht zu erstatten, welchem wir zunächst unsere Aufmerksamkeit widmen wollen.

Papst Pius II., so vernehmen wir, hielt am 2. unil 15. September ${ }^{3}$ ) 1459 Beratungen mit den burgundischen Herren ab, und zwar allein mit ihnen, ohne dass Gesandte der anderen Herrscher zugegen waren ${ }^{4}$ ). Mit eiuem recht stattlichen Programm trat der Papst herror: er verkündete, ein Heer von 80000 Reitern und Fusssoldaten, sowie eine Flotte von 60 oder 50 oder wenigstens 35 Galeeren und 20 gerüsteten Fahrzeugen zu stellen und hoffte von Philipp, den ja sein Vorgänger Kalixt III. als deu , christianae fidei fortissimus athleta et intrepidus pugil* gefeiert hatte ${ }^{\mathfrak{j}}$ ), feste Zusagen für deu Kriegszug zu erhalten.

France avec l'Allemagne de 1378 à 1461 (Paris 1892) kennen unser Stück nicht; auf' die Brüsseler Hs. wies flüchtig Kervyn de Lettenhove, Hist. de Flandre (Bruxelies 1850) 47 hin und nach ibm P. Eredericq, Essai sur le rôle politique et social des ducs de Bourgogne dans les Pays.Bas (iiand 1875) 45.

1) Hanneron wirkte auch an der Erziebung Karls des Kühnen wit; vgl. den Zahlungsbefehl an , Ant. Hanneron ... maistre d'escolle de monseigneur le conte de Charolais ', Lille Arch. du Nord B 1978 t. 53. Bei John Foster Kirk, Hist. of Charles the Bold I (London 1863) $108 \mathrm{ff}$. sehe ich nichts davon erwähnt.

2) Vgl. L. Pastor, Gesch. der Päpste II s u. + (Freiburg i. Br. 1904) $57 \mathrm{ff}$. Wie Mathieu d' Esconchy ed. du Éresne de Beaucourt, Soc. Hist. France II (Paris 1863) 382 mitteilt, nahm Hanneron auf dem Prunkmahl, das Franz Sforza in Mailand zu Ehren der burgundischen Gesandten gab, einen bevorzugten Platz in der Nähe der Herzogin ein.

s) Diese Daten sind von Wichtigkeit, da die Ankunft der burgundischen Gesandten in Mantua nicht genau feststeht. Denn auch die Angaben ron Matthieu d' Escouchy 1.1. If $385 \mathrm{ff}$., auf die wir hier allein angewiesen sind, treffen nicht genau zu. Der Freitag, an welchem die Gesandten in ,Brugelle eintrafen, fiel (was Pastor Il 57 Anm. 5 entgangen ist), nicht auf den 15., sondern auf den 17. August. Am Sametag [18. August] lässt der Chronist die Gesandten 3 oder 4 Miglien vor Mantua sein, berichtet dann vom feierlichen Einzuge, von der Audienz am, folgenden Mittwoch • [22. August] und spricht dann plötalich von der Messe, die der Papst, ile jour Notre Dame my aoust ensievant abhält.

4) Somit schlug der Yapst dies Verfahren nicht erst nach der offiziellen Eröflnungssitzung vom 26. September ein; (i. Voigt, Enea Silvio de'Piccolomini als Papst Pius Il. und sein Zeitalter III (Berlin 1863) 73 und Pastor IL 66.

5) In der Bulle, Dum praeclara longe lateque per orbem " rom 9. Jan. 1454; Org. Brüssel, Arch. Gén., Chartes de la Clambre des Comptes nr. 234\%. Vgl, auch Pastor I 607 Anm. 5. 
Aber erst nach längerem Zögern entschlossen sich die Gesandteu dazua: 4000 Mann zu Fuss uud 2000 Reiter zu bewilligen, womit der Papst durchaus nicht so zufrieden war, wie es hier dargestellt wird ${ }^{1}$ ). Musste er docb noch Philipp für die Kosten des Heeres entschädigen und ihm die Zehnt und Indulgenzerträgnisse in den burguudischen Landen zusicheru ${ }^{2}$ ).

Nachdem diese Abmachungen am 12. September getroffen warew, reiste die Mehrzahl der burgundischen Gesandteu $a b^{\mathfrak{3}}$ ); Nagister Hanneron begab sich mit Simon de Lalaing. der bereits häufig sein Geschick bewährt hatte. an deu Hof Kaiser Friedrichs.

In ihrer Mission handelte es sich vornehmlich um vier Dinge.

1. Um die Leistung des Eides für die im Besitz des Burgunder: befindlichen Reichslehen. Philipp batte Kaiser Sigmund die Huldigurg verweigert und war daher iw J. 1434 zum Reichsfeind erklärt worden ${ }^{*}$. Niit Friedrich gingen seit $1442^{5}$ ) Verhandlungen, die 1447 beinahe $z u$ einem Ergebnis geführt batten ${ }^{6}$ ) und jetat ron neuem aufgenommen wurden. erwäbnt.

1) Vgl. sein Schreiben vom 16. September 1459, das Pastor 1158 Anm. 1

") Daron war noch nichts bekannt. - Vgl. dazu A. Gottlob, Aus der Cameria Apostolica des 15. Jahrhunderts (Innsbruck 1889) 181.

3) Johann von Klere, der an der Spitze der Gesandtschaft stand, hatte au der Kurie auch persönliche Angelegenbeiten betrieben; vgl. J. Hansen, Westfalen und Rheiuland in 15. Jahrhundert II (Leipzig 1890: Publ. aus den Preuss. Staatsarchiven Bd, 42) 138*. Für seine Reiseunkosten erhielt er vom 19. Juni bis zum 16. September die Summe von 7200 Pfund; Lille, Arch. du Nord. B 2034 Compte de l'année 1459 f. $95^{r}$, wo auch die Auggaben für die anderen Gesandten angegeben sind. - Vgl. auch Pastor II 58 Anm. - Der Herr von Croy besuchte auf dem Rückwege das Kastell des Sforza in Pavia; vgl. C. Magenta, I Visconti e gli Sforza nel castello di Pavia I (Pavia 1883) 456 Anm. 4.

Die Anwesenheit burg. Gesandter in der Sitzung voun 26. Sept. wird in Pii Secundi ... Commentarii etc. (Francofurti 1614) 82 erwähnt.

4) Deutsche Reichstagsakten XI nr. 287: H. Pirenne, Hist. le Belgique 11 (Bruxelles 1903) 228 ff:

5) A. Perier, Nicolas Rolin 1380-1461 (Paris 1904) 256 (dann auch S. 293) behauptet, dass man in der Zusammenkunft Friedrichs mit Philipp zu Besançon im Jahre 1442 , die Verzichtleistung der Rechte des Kaiserreiches auf Holland, Seeland und Brabant erreichte ${ }^{\circ}$ und beruft sich auf Olivier de la Marche 1 (ed. Soc. Hist. Fr.) 279, der davon aber gar nichts sagt, sondern nur die Verhandlungen in dieser Sache erwähnt. Vgl. Chmel, Geschichte K. Friedrichs II $245 \mathrm{ff}$. D.e irrige Nachricht geht wohl zurück auf Pontus Heuterus, Rerum Burgundjcarum libri VI (Hagae-Comitis 1639) 288.

ค) Vgl. die für Philipp ausgesteilten, aber nicht ausgefolgten Lehensbriefe vom 20. Sept. 1447 (Chmel, Regesta . . Friderici [II. nr. 2330 u. 2331) und den 
2. Um die Luxemburger Frage, die vor kurzem in ein neues Stadium getreten war. Am 20. März 1459 hatten Herzog Wilhelm von Sachsen und seine Gemahlin Anna, die älteste Tochter König Albrechts und Schwester des früh rerstorbenen König Ladislaus', das Herzogtum Luxemburg, das Philipp seit 1443 als Pfandherr besass, mit allen Rechten an König Karl VII. verkauft ${ }^{1}$ ), wodurch die bereits bestehende Spannung zwischen Frankreich und Burgund noch bedeutend vermehrt wurde:). Nachdem im September 1459 Philipp gegen deu Verkanf protestirt und seinem Gegner erklärt hatte, die Abtretung des unzertreunlich zur Krone Böhmen gebörigen Herzogtums sei ungiltig und könne nur mit Genehmigung des Kaisers geschehen ${ }^{3}$ ), versuchte er diesen jetzt persönlich für die Saclie, an der auch ein Widersacher Friedrichs, Georg Podiebrad, beteiligt war, zu interessiren und eine günstige Eutscheidung herbeizuführen.

3. Cm das "Vikariat". Bereits 1447 bei der Sendung Heinrichs ron Heessel uach Wien war von Philipp im Zusammenhang mit der Erhebung des burgundischen Besitzes zum unabhängigen Königreich "eine Art von Vorherrschaft über die in Niederdeutscbland gelegenen Territorien ${ }^{4}$ ) gefordert worden, worauf jetzt zurückgegriffen wird.

4. Üm ein Bündnis zwischen Friedrich und Philipp.

Wie früher, so führten auch im Oktober 1459 die Verhandlungen zu keinem Resultate; der Kaiser bat sich Bedenkzeit aus und beraumte auf den 1. Mai 1460 eine neue Besprechung an, welcher am Sonntag Judica (30. März) der vom Papst festgesetzte Türkentag vorhergehen sollte.

vorgeschriebenen Lehnseid (Chmel, Materialien zur österr. Gesch. II 277 nr. 120). Auch F. Rachfahl, Die Trennung der Niederlande vom deutschen Reiche, Westd.Leitschr. f. Gesch. u. Kunst 19(1900) 83 wies gegen P. J. Blok, Geschiedenis ran bet nederlandsche Volk II (1893) 273 [deutsche Ausgabe 1903 S. 337] darauf hin, dass Philipp im Jahre 1448 keineswegs die Belehnung mit den Reichslehen erhielt, was auch Leroux l. l. 210 falschlich behauptet.

1) Vgl. N. van Wervecke, Defnitive Krwerbung des Luxemburger Landes durch Philipp, Herzog von Burgund (Lux. 1886; Sonderabzug aus den ,Lux. Land.' 19.

2) In dem unten S. 452 Anm. 2 erwähnten Schreiben heisst es: [Hanneron] ne ha poi dicto che ha lassato le cose tra la maesta del re di Franza et dicto suo signore molto turbolente e quasi tutte disposte ad guerra ... et questo procede principalmente per tre sose quale rechiede el prefato re ad esso duca ... la seconda qui le voglia restituere el ducato de Lucimbergo.

ง) Dufresne de Beaucourt l. l. VI $278 \mathrm{ff}$ :

๑) V. v. Kraus, Deutsche Gesch. im Ausgange des Mittelalters I (Stuttgart u. Berlin 1905) 282; $\nabla g l$. die Instruktion für Heinrich von Heessel im ,Österr. Geschichtsforscher" bg. ron Chmel I 235. 
Philipp gedachte wieder deaselben Gesandten die Aufyabe anzuvertrauen; aber Simon de Lalaing erkrankte, und schliesslich nusste sich Hanneron allein auf den Weg machen ${ }^{1}$ ), nachdem beide Terunine verstrichen waren. Dann trat noch eine neue Verzögerung ein. da Hanneron infolge des Kampfes zwischen dem Markgrafen Albrecht Achilles rou Brandenburg und den Wittelsbachern nicht durch Süddeutschland reisen, sondern den beträchtlichen Umweg über Mailand machen musste ${ }^{2}$ ). Alber trotz allen Aufschubes traf er noch rechtzeitig genug in Deutschlaud ein, um am 17. September in Wien den Türkentag unter dem Vorsitze des Kardinallegaten Bessarion mitzumachen; denn so ärgerlich es auch dem vor Eifer glühenden, seiner Heiuat beraubten Griechen gewesen war, die beiden vou Papst Pius in Mantua einberufenen Versammlungen hatten wegen der erwähnten kriegerischen Unruhen noch nicht stattfinden können.

Hier in Wien wiederbolte Hauneron - denu sicherlich ist er der in den Protokollen aufgeführte ,orator ducis Burgundiae*3) - die auf den Mantuaner Kougresse gegebene Zusage. Dann galt es, nachdem er dem Kaiser über die dort mit dem Papste getroffenen Abmachungen Bericht erstattet hatte ${ }^{4}$, die ungefähr vor Jahresfrist abgebrochenen Verhandlungen wieder anzuknüpfen.

Ausfübrlich soll Hanneron die Rechte Philipps auf die Reichsiehen auseinandersetzen. Auf die alten Chroniken wird hingewiesen, die Nachricht geben über das schöne und weite Königreich „Lothier", das jederzeit sein eigenes Recht in Lehenssachen gehabt habe, das sich anstandslos auf Frauen und deren Nachkommen vererbte, wie durch Beispiele erhärtet wird.

Und noch ein wirksameres nittel als diese gelehrte Auseinandersetzung wird in der Instruktion nicht vergessen: reiche und klingende Belohnung darf der Gesandte dem stets knappen und knauserigen Kaiser in Aussicht stellen, was bei des Burgunders weit und breit bekannten Schätzen besonders verlockend war.

1) Gemäss einer interessanten Zusammenstellung der Einnabmen und Ausgaben für Philipps, voiage de 'l'urquie (die ich in Lille fand und demnächst veröffentlichen werde), erhielt Hanneron für die Reise an cien Hof des Papstes, des Kaisers und der Signorie 1£08 Pfund.

2) Hierbei waren sicherlich auch politische Gründe mit im spiele. -- In einem Schreiben Franz Sforzas an Anton Guidoboni in Venedig vom 9. Jun: 1460 finde jch Hannerons Ankunft in Mailand am 8. Juni erwähnt; Mailand, Arch. di Stato, Pot. Estere, Venezia. Den Beziehungen zwischen Burgund unc Italien im 15. Jb. gedeuke ich eine besondere Studie zu widmen.

s) Vgl. H. Chr. Senkcnberg, Selectal juris et historiarum IV 366.

1) s. oben $\mathrm{S}, 449$. 
Fom Kaiserhofe aus, an welchem Hanneron wenn nützlich auch noch die "manière d'aliance que l'on scet" in Anregung bringen konnte, soll er sich nochinals zum Papste begeben und ihm, der den Königspläuen des Herzogs woblwollend gegenüberstand, üher die Ergebnisse Mitteilung nachen und - auch eine Reihe von Bitten ${ }^{1}$ ) vortragen. Philipp wusste wie notweudig seine Hilfe dem Papste war, wenn es zu dem Kreuzzuge gegen die Türken kam, der seit der Eroberung Konstantinopels die Kurie in Atew hielt und ihn selbst bereits seit langen Jahren beschäftigte 2 ).

Instruction pour maistre Antboine Hanneron, docteur en décret prothonotaire du Saint Siège apostolique, arcediacre de Cambray, prévost des églises de Mons, conseiller de monseigneur le duc de Bourgogne et maistre des requestes de son hostel, lequel matistre Anthoinne mondit seigneur le due envoie devers l'Emperear premièrement, et de là devers nostre Saint Père le Pape. do ce que ledit maistre Anthoinne aura a faire pardevers eulx ${ }^{3}$ ).

Premièrement, ledit maistre Anthoinne lui venu devers l'Empereur $s^{\prime}$ adressera devant le cambremeister l'évesque de Curse et messire Wylric ${ }^{4}$ ), leur baillera les lettres de mondit seigneur à eulx adressans contenans ereance sur ledit maistre Anthoinne, pour laquelle créance il leur déclairera la charge qu'il a de parler et besongnier devers l'Empereur, les requerra de leur ayde et adresse pour avoir accès et audience devers luj et leur dira que pour la conduitte de sa charge et de tontes les matières pour lesquelles mondit seigneur l'envoie devers l'Empereur; mondit seigneur a sa plainière et entière confidence en enls et en leur bon moien et que comme autresfoiz messire Simon de Lalaing, seigneur de Montigny, et ledit maistre Anthboinne leur ont dit, leur peirne et traveil seront bien recongneuz et n'y aura quelque faulte en ce qu'ilz leur en ont dit et promis, en leur priant que ilz y vueillent telement faire que à ceste foiz pour toutes, conclusion soit prinse sur lesdictes matières.

Item, quand ledit maistre Anthoinne aura accès devers l' Emperear pour la première foiz il fera les recommandacions denes et accoustumées

1) Ich kann auf die einzelnen nicht näher eingehen, da unir augenblicklich die Lokalliteratur nicht zur Verfügang steht.

₹) Über Philipps Kreuzzugspolitik hoffe ich bald eine Untersucbung zu reröffentlichen.

3) Auf dem Unschlag steht: Instructions de monseigneur le duc Philippe, cuy Dieu pardoint, à maistre Anthoine Hanneron pour aler devers l' Empereur pour le fait du vonaige de T'urquie et les terres, pays et seignories que icellui seigneur tenoit soubz l'Empire.

4) Diese Persönlichkeiten kann ich nicht mit Sicherheit bestimmen. Der ,évesque de Curse * ist wohl Bischof Ulrich von Gurk, der österr. Kanzler; der ,messire Wylrie Ulrich Riederer, Dompropst von Freising und römischer Kanzler. 
pour mondit seigneur, lui baillera ses lettres contenans créance sur lui et pour icelle sa créance dira et exposera ce qui s'ensuit:

Assavoir, que mondit seigneur l'a envoié devers sa Jagesté Impeirial pour deux causes. l'une pour satisfaire et fournir de sa part it lit journée prinse pardevers lui touchant le secours et ayde contre le Turc. ainsi qu'il avoit pleu is sadicte Magesté escrire et signiffier à mondit seigneur $q u$ 'il fust ou envoïast à icelle journée, l'autre pour saroir son bon plaisir et avoir sa response sur les matières pour lesquelles ledit messire Simon de Lalaing et lui furent ou mois d'octobre derrenièrement liasié enroiez devers lui de par mondit seigneur, et surquoy il pleust à sadicte Magesté prandre delay pour soy sur tout faire mieulx informer en assignant ceste présente journee pour en bailler à ceulx que mondit seigneur le duc envoieroit sa response. Et ja soit ce que la journè touchant la première matière fust ordonée estre tenue dès le dimenche Judica '), et l' autre au premier jour de may, toutevoies mondit seigneur avoit tardé d's enroïer son ambassade pour ce que pour certainnes causes et considéracions il vouloit envoïer à ceste journée ceulx mesmes qui derrenièrement avoient esté devers sadicte Magesté, assavoir ledit messire Simon de Lalaing et ledit maistre Anthoinne sans en baillier charge à autres qui par cidevant n'en ont eu quelque entremise: or est avenu que ledit messire Simon a long temps esté fort malade d'une jambe. comme il est encores de present. et telement qu'il n'a peu emprandre le chemin et a l'on attendu pour lui soubz espoir de sa garison tant que le temps s'est grandement passe et finablement. a convenu que ledit maistre Anthoinne ait prins ceste cbose seul et quant il a cuidié prandre son chemin par les Alemaignes, il a en chemin esté allverti de la grant tribulacion de guerre qui y règne présentement, et pour quérir seurté lui a convenu faire grant circuite par Lombardie de là les mons où il a emplor̈é grant temps en excusant par ce la tardiveté d'estre venu à icelle journée, tant pour mondit seigneur le duc, comme aussi pour ledit maistre Anthoinne.

Item, el pour venir au propoz desdictes matières, premièrement touchant le fait du Ture dira ledit maistre Anthoinne, comment après pluseurs journées et assemblées tenues sur ladicte matière ès paìs de Hongrie, d'Alemaigne et ailleurs finablement nostre Saint Père le Pape considérant que desdictes journées et assemblées ne s'estoit ensuy aucun fructueulx effect, advisa et conclu arec messeigneurs du Saint Collège de tenir certainne journée en la cité de Mantua ou mois de juing derrenièrement passé et de $\mathrm{y}$ convoquer et assembler les princes chrestians qui vraisemblablement pevent faire ayde et confort à la sainte Foy contre l'outrageuse emprinse du Turc, en prenant et recueillant toute ceste haulte matière en sil main comme chief et prince universel de toute la chrestianté, et à laquelle journée et convencion les princes chrestians ont fait leur devoir d'envoïer chascun en droit soy et ont illec oy ce qu'il a pleu à nostre dit Saint Père leur dire et proposer touchant ceste matière et en ont besongnié avec sa Saincteté en accordant et déclairant ce qu'ilz vouloient ou povoient faire d'ayde et de secours en ceste partie, nos pas d'une voix commune, ne en assemblée des ungs avec les autres, mais chascun pour soy: ainsi

り 1460 März 30 . 
qu'il pleu à nostre dit Saint Père prandre et eslire la voie et manière de besongnier arec eulx; et entre autres mondit seigneur le duc y envolia yrant et moult notable ambassarle: assavoir monseigneur le duc te Clèves, son neveu, accompaignié de évesques et prélatz ecclésiastiques, de barons, chevaliers et autres docteurs et gens de conseil en grant nombre: lesquelz attendirent au dit lieu de Mantua bien longue espace de temps pour la venue des autres princes ou de leurs ambassadeurs, affin d'avoir adviz et communication ensemble, et finablement, après bien longue attente aux gran; fraiz et despens de mondit seigneur, véuns que nul ne se hastoit de venir et par l'advis de nostre Saint Père, besongnèrent devers Sa Saincteté et messeigneurs du Saint Collège en leur déclairant ce que mondit seigneur, selon la disposicion de ses affaires et de ses paîs povoit et vouloit faire d'ayde et de secours pour mettre sus et soustenir une armée contre le Turc, en faisant de ce offre à nostre dit Saint Père pour celle foiz. Et de laquelle offre nostre dit Saint-Père et mes disseigneurs les cardinaulx se tindrent pour très bien contens, tet sur ce se départi ladicte ambassade dudit Mantua et print congié de nostre dit saint Pere et de mesdisseigneurs. Et depuis, pour esmouvoir les autres princes ou leurs ambassadeurs qui survindrent à icelle journée, fist nostre dit Suint Père icelle offre de mondit seigneur dire et réciter en consistoire publique, affin que chascun y print pie commandement et exemple de quelque chose offrir et présenter pur ceste saincte besongne, comne depuis les pluiseurs s'en sont haultement et loablement acquittez et mesmement sadicte Magesté Impérial, comme mondit seigneur a depuis esté bien acertenez.

Item, et l'offre que firent lors les ambassadeurs de mondit seigneur fut tele qu'il s'ensuit: Sur ce qu'il a pleu à nostre Saint Père le Pape dire à très-hault et puissant prince monseigneur le duc de Clèves et aax ambassadeurs de monseigneur le duc de Bourgoingne et de Brabant estans en ceste convencion de Mantua touchant l'expédicion et armée que nostre Saint Père entendoit à mettre sus pour résister contre la puissance du Turc, ennemi et persécuteur de la religion chrestianne, et laquelle armée nostre dit Saint Père entendoit estre de quatre vins mil combatans de pié et de cheval conduiz par terre ou royaulme de Hongrie et par mer d'autre nombre de combatans: c'est assavoir de soixante, cinquante ou au moins de trente cinq galées et de vint nefs armées pour commancier la guerre contre ledit Turc en arril ou en may pronchain venant à l'ayde des princes chrestians. Et sur ce que nostre dit Saint Père a requis à mondit seigneur de Clèves et ausdits ambassadeur's qu'ilz, plus particulièrement et autrement que fait n' aroient, voulsissent déclairier quel ayde et quel nombre de gens mondit seigneur de Bourgoingne vouldroit faire pour aydier à fournir ladicte armée tant par mer que par terre, et pour icelle armée continuer et entretenir se mestier estoit en la prouchainne saison d'esté et en autres deux semblables saisons ès deux années suivans. Et sur ce aussi que nostre dit Saint Père a ouvert que pour faire laılicte armée et l' entretenir comme dit est, et afrin que les fraiz soient a la charge d'un chascun supportez également, que l'on mettra sus et pourra l'on lever durans lesdictes trois années ung décime sur toutes gens d'église et le $X^{\prime} X^{\ominus}$ denier de toutes les rentes et revenues annuelles de tous les gens laiz et dont les deniers seroient receuz et distribuez par ceulx qui seroient à ce commis 
par nostre dit Saint Père et par les princes et par le clergié de chascum païs on province; et pour à ce mieulx parvenir et avoir le consentement desdits lays, nostre dit Saint Père le Pape donroit indulgences plenièress à tous ceul $\mathbf{x}$ qui bailleroient pour ung an ledit $\mathbf{X X X ^ { \theta }}$ de leursdictes rente.s et revenues tant seulement; offrant nostre dit Saint Père à mondit seigneur de Bourgoingne lui laissier les prouffiz qui vendroient lesdits trois ans durans desdits décimes sur lesclictes gens d'église et dudit $\mathrm{XXX}^{\theta}$ sur lesdits lays et aussi de toutes indulgences en sesdits pais, terres et seigneuries et enclaremens d'icelles pour le tout convertir et emploier ou fournissement de ladicte armée. A esté par mondit seigneur de Clèves et par mesdis seigneurs les ambassadears respondu et remonstré a nostre dit Saint Père, en la présence de pluseurs de messeigneurs les cardinaulx. comment par leur première response par eulx faicte le $\mathrm{II}^{\theta}$ jour de ce mois ilz avoient déclairé le grant et bault vouloir et l'ardent désir que mondit seigneur cle Bourgongne avoit tonsjours eu et avoit à soy emploïer en ceste matière et comme selon la teneur da veu par lui fait, se les condicion. y contenues estoient ou povoient estre accomplies, il y vouldroit emploïer sa personne et toute la puissance terrienne que Dieu, p.r sa grâce, lui a donné en cestui mortel monde. Et ont aussi remonstré comment pour faire une grant ayde générale en laquelle tous les princes chrestians puissent eulx emploïer pour résister à la puissance dudit Turc: il senbloit que nostre dit Saint Père se devoit emplö̈er à l'appaisement de tous les princes et communaultez qui avoient guerres ou divisions, et que se neantmoins ledit appaisement ne se povoit prestement faire ainsi que autresfoi\% avoit esté remonstré à nostre dit Saint-Père, lequel alors déclaira que son intencion estoit de mettre sus une armée de quarante ou cinquante uil combatans oultre et pardessus la puissance du royaulme de Hongrie et des païs voisins dulit 'Turc, il estoit vray que mondit seigneur de Clèves, pour et ou nom de mondit seigneur de Bourgoingne, son oncle, avoit offert de aydier a fournir ladicte armée et $y$ faire arecques les aultres princes et communaultez, si avant et telement qu'il apparroit is ung chascun, que à lui ne tendroit que la religion chrestienne ne fust deffendue et que pour arrester la part et porcion de ladicte armée et dont demouroit chargié mondit seigneur de Bourgoingne, et aussi pour adviser comment et quant se mettroit sus icelle armée, fut dit audit $\mathrm{II}^{\mathrm{t}}$ jour de cedit mois que mondit seigneur de Clèves laisseroit aucuns desdits ambassadeurs qui demourroient pour ceste cause de lez nostre dit Saint Père en at.tendant ln venue des autres princes et de leurs ambassadeurs ${ }^{1}$ ), et que considérez les grans affoires de mondit seigneur le duc, l'estat et disposicion de ses paîs, il sembloit que ladicte offre estoit belle, souffisant et honneste et de laquelle nostre dit Saint Père se devoit contenter, combien qu'elle fust en terme généraulx, veu que aucuns antres princes n'avoient encores riens offert en espécial, ne riens dit plus avant que avoit mondit seigneur de Bourgoingne: Tontevoies pour ce que nostre dit Saint Père ne s'estoit voulu contenter d'icelle offre générale, ne aussi de certaine autr't offie à lui plus particulièrement faicte et qu'jl a déclairié sa voulenté plur particulièrement tant du nombre des combatans, comme du temps et des lieux on se fera la guerre, que il n'avoit encores fait, et aussi qu'il s

1) s. oben S. $450 \mathrm{Anm}$. 3. 
dit que pour le lien et l' effect de ceste convencion, il a eu tousjours sa confidence et sen espoir en mondit seigneur de Bourgoingne et en l'offre que mondit seigneur fist a Franquefort; mondit seigneur de Clèves et lesdits aubassadeurs après pluseurs remonstrances faictes à nostre dit Saint Yère tant de ladicte journée de Franquefort ${ }^{1}$ ) comme autrement lui ont dit que se ladicte armée de IIIIXX mil combatans se met sus par terre oudit royaulme de Hongrie en la prouchainne saison d'esté que en ce cas mondit seigneur de Bourgoingne souldoiera quatre mil d'iceulx combatans de pié et deux mil combatans à cheval durant ledit temps et saison d'esté que ladicte armée sera sur les champs tant sealement et non plus avant, pour résister, combatre ou adommagier ledit Turc. Et au regart de l'entretenement de ladicte armée après ledit temps et saison de l'esté fini, et anssi de la continuer ès antres denx années ensuivans comme dit est, et pour quoy faire nostre dit Saint Père a requis que mondit seigneur de Bourroingne consente que ledit XXXe soit mis sus sur les lays en sesdits pays et enclavemens ainsi qu'il avoit intencion de le requérir à tous les autres princes et qu'il aroit espoir de l'obtenir de tous, ainsi que desjà aucuns l'avoient accordé et consenti; mondit seigneur de Clèves et lesdits ambassadeurs ont dit et remonstré les affaires de mondit seigneur de Bourgoingne et comment la charge d'entretenir et de continuer ladicte armée plus avant que d'une saison estoit grande et de grans fraiz, et aussi que la manière et lis voie ouverte c'est assavoir de mettre sus ledit XXXe sur lesdits lays estoit chose moult nouvelle et dont il estoit besoing que mondit seigneur de Bourgoingne fust premiers adverti. Et ponr ce ont requis et obtenu de nostre Saint Père délay pour en faire rapport à mondit seigneur et se sont chargiez de certiffier nostre dit Saint Père de la vonlenté de mondit seigneur le plustost que bonnement pourroit durant ceste présente convencion; mais néantmoins touchant ledit décime pour le lever en ung an sur lesdictes gens d'église esdits païs et seigneuries de mondit seigneur et ès enclavemens, ainsi et par la forme et manière que l'accorda autrestoiz à mondit seignear de Bourgoingne fen pape Nicolas, oultre et pardessus les autres aydes et décimes que mondit seigneur a levées et qui lui sont accordées pour aydier à soustenir les fraiz qu'il fera pour ladicte armée: laquelle offe, jceulx ambassadeurs avant leur dicte response faicte et depuis ont accepté, et aussi ont accepté ce que nostre dit Saint Père a offert it mondit seigneur de Bourgoingne de baillier et ottroier indulgences plenières en sesdits paìs et seigneuries, qui dureront trois ans entiers, du proufit desquelles mondit seigneur aurd le tiers oultre lesdits décimes pour l'ayde qu'il fera en ceste pronchainne saison se ladicte armée se met sus comme dit est et les autres deux tiers desdits proufiz d'icelles indulgences seront gardez pour les emploier en l'expédicion et en l'armée qui se fera et se continuera ès autres années ensuivans. Ce fut fait et conclud à Mantua le XVe joui de septembre l'an mil CCCC cinquante neuf, présens et assistans avec nostre dit Saint Père, très-révérends pères en

1) Vgl.Voigt 1. 1. 119 ff.; in dem Truppenanschlag wird der Herzog von Burgund nit 3000 Mann zu Pferd und 6000 Mann zu Fuss angeführt; vgl. Gustav Gieorg Königz von Königsthal Nachlese in den Reichsgeschichten 1 (Franturt a. M. 1759) 34. Die Speier. Chronik (Mone, Quellessammlung der Badischen Landesgeschichte 1 397) gibt 2000 Mann zu Pferd und 4000 Mann zu Fuss an. 
Dieu, messeigneurs les cardinaulx de Nicene ${ }^{1}$ ), de Ronen ${ }^{2}$ ), des Crsins $^{3}$ ), $d^{\prime}$ Ostun ${ }^{4}$ ), d'Avignon ${ }^{5}$ ), de Boulongne ${ }^{6}$ ), et de Zamorensis ${ }^{7}$ ).

Item, et laquelle offre mondit seigneur a tousjonrs depuis eu roulenté et propoz de faire et fournir et encores est wondit seigneur de cestre voulente et intencion et $n^{\prime} \mathrm{y}$ aura, se Dieu plaist. faulte de sa part iqu'il ne la parfournisse ainsi et par la manière comme il a esté dit et offer't a nostre dit Saint Père et cy-dessus récité.

Item, et touchant la $\mathrm{II}^{\mathrm{e}}$ cause de ceste journee, leilit maistre Antbuidr diri comment sur les matières que ledit messire Simon de Lalaing̣ et lui comme ambassadeurs de mondit seigneur on mois d'octobre derrenitrement passé proposèrent à sadicte Magesté Impérial de par mondit seigneur touchant les hommages et infeudacions des terres et seigneuries que mondit seigneur tient de l'Empire et certainnes autres matières dont il sulpose assez sadicte Magesté avoir bonne souvenance, il pleust à icelle sa Migesté prandre délay pour $y$ penser et ordonner et assigner ceste journée pour en baillier sa response et demanderi icellui maistre Anthoinne ladicte response.

Item, et se l'Empereur vouloit que ledit maistre Antboine raffireschist et répétast icelles matières, il le fera le plus brief qu'il pourra. en touchant les quatre poins le ladicte première ambassade: assavoir des howmages des duchien, comtez, terres et seigneuries que mondit seigneur tient de l'Empire, du don du tiltre du duchié de Luxembouro que l'Empereur y puet prétendre ou au moins confirmacion des gagières que mondit seigneur a sur ledit duchié, du povoir ou commission sur les marches de deçà le Rin pour garder et soustenir les droiz impériaulx etc. ${ }^{2}$ et de aliance faire et conclurre entre l'Empereur et mondit seigneur pour euls, leurs subgez, terres et seigneuries, en concluant tousjours pour avoir la response de l'Empereur sur cbascun desdits poins et articles.

Item, et ou cas que de par l'Empereur fust bailliée ladicte response, ledit maistre Anthoinne mettra peinne de l'avoir par escript et quant il l'aura ainsi recouvrée ou que lui mesmes l'aura rédigee et recueillie par escript à son entendement et monstré au conseil de l'Empereur, tant que l'on si puist seurement arrester, lors, dira à l'Empereur comment il il advisé de l'envoïer bien et diligemment par propre messagé devers monseigneur le duc pour en savoir son bon plaisir et ne bougera dudit lieu de devers l'Empereur jusques à ce qu'il en ait et sache le plaisir de mondit seigneur pour, au surplus, en faire et poursuir ainsi qu'il lui sera par lui commandé et rescript.

Item, et ou cas que l'Empereur avant qu'il baillast sa response voulsist avoir plus ample déclaracion desdictes matières, mesmement touchant le premier point des investitures et hommages des terres et seignouries que mondit seigneur tient de l'Empire, assavoir se mondit seigneur se voul-

"Bessarion.

$\because$ Wilhelm d'Estouteville.

3) Latinus Orsini.

4) Johannes Rolin, Bischof ron Autun.

5) Alanus de Coetivy.

i) Philippus Calandrini, Bischof ron Bologna.

') Johannes de Mella. 
1roit contenter de lettres en termes générauls, sans riens spécifier, assavoir de tuutes terres et seignouries, duchiez, ou comtes et autres quocumque nomine censeantur ainsi que en la derrenière ambassade de mondit seigneur envoiée devers sarlicte Magesté fut requis et demandé, ou s'il vouldroit aroir la déclaracion expresse de bien et deuement avoir succedide esdictes terres et seignouries par noïen de femme et en ligne collatécial, et combien l'on vouldroit offrir de récompense à l'Einpereur pour l'une et l'autre matière. Item, et touchant le fait de la donacion du tiltre du duchié de Luxembourcy quelle récompense pour l'Empereur ou pour la confirmacion des lettres de la gagière. Itero, et sur la commission du vicariat, aussi quelle récompense etc.: icellui maistre Anthoinne pour le don du tiltre de Lurembourg après pluseurs remonstrances qu'il n'y cbiet pas grant récompense etc. ${ }^{a}$, pourra finablement offrir jusques a la somme de dix unil florins de Rin, et ou cas qu'il ne peust obtenir le clon dudit tiltre, pourra oftrir pour la confirmacion de ladicte gagière ........ ').

Item, et touchant le vicariat il pourra offrir le tiers et finablement la moittié de tous deniers qui en venroient a mondit seigneur. et sur le surplus mondit seigneur supporteroit les despens qu'il feroit en l'exercice d'icellui vicariat. Item, et touchant lesdits hommages il differera de riens léclairier de récompense tant qu'il sache préalablement quelle chose l'en lui vouldra faire sur ce, et ainsi contendra à différer et reculer au mieulx qu'il pourra pour tousjours gaignier temps, tant quil ait ladicte response, pour la lesurire pardeça comme dit est dessus et en attendre ce que mondit seigneur lui mandera.

Item, et ou cas que touchant lesdits investitures et hommages, soit demandé audit maistre Anthoinne de bailler par escript à l'Empereur sa demande avec toute déclaracion des droiz où il vaelt fonder le fait de mondit seigneur, attendu que le droit commun des fiefs impériaulx est tout contraire, et fault bien que mondit seigneur se fonde en quelque fait espécial de privilège s'il vuelt venir à son intencion et aussi qu'il baille par déclaracion la généalogie et descendue de mondit seigneur pour veoir et entendre par quel degré de consanguinéité il entend devoir succéder ausdictes terres et seignouries devant tous autres et comme le plus prouchain héritier: dira ledit maistre Anthoinne que la nature et condicion desdictes terres et seignouries et autres voisinnes de deçà le Rin, commo Ghelres, Clèves, Lorrainne et antres si est et a tousjours notoirement esté en franchise de succéder au plus prouchain sans distinction de masle ou de femme et en ligne collatéral en deffault de ligne directe et ne sera jamais trouvé que esdictes terres et seignouries femmes aient esté deboutées et fourcloses de la succession quant elles ont esté trouvées en plus prouchain degré pour y succéder, ne que jamais à leur cause droit le dérolucion à l'Empire $\mathrm{g}$ ait eu lieu par faulte de hoir masle, et n'est pas aussi de merveille que ainsi soit et que lesdictes seigneuries de deçà le Rin ne soient quant à ce de tele servitude comme celles de delà le Rin, car l'on treuve par anciennes croniques que l'Empire ne souloit s'extendre que jusques au Rin et entre le Rin et le royaulme de France estoit ung royaulme bel et grant contenant pluseurs belles et grandes villes

1) Freigelassen. 
et citez que l'on nommoit le royanlme de Lothier, et treuve l'on que le royaulme de Lothier estoit ung royaulme scitué entre l'Escaut et le Rin et entre Bourgoingne et la mer de Frise ouquel royaulme sont et $y$ a trois églises métropolitainnes, assavoir: Mayance, Trèves et Coulongne et les cathédrales qui s'ensuivent, c'est assavoir: Mets, Toul, Verdun. Cawbras, Liège et Utrecht et à cause de Lothaire premier roy fut ledit royaulme appellé Lothier; mais icellui royaulme n'eut pas yrant durée, car les roys de France commancèrent de leur costé à emprandre et attribuer certainne porcion à leur royaulme et les empereurs d'Alemaigne tirèrent d'autre costé tant que ledit royaulme fut tout desseuré et en vint la pluspart a l'Empire ou elle est encores de présent; mais si ont les terres et seigneuries d'icellui tousjours retenu leur nature et condicion ancienne touchant ladicte manière de succession, non pas pour les principaultez seulement, mais aussi quant aux fiefs des vassaulx mourans desdictes seigneuries, lesqielx succèdent tous et ont tonsjours notoirement succedé sur l'un et l'autre sexe et par ligne collatéral en faulte de ligne directe, et est chose si notoire par lesdictes marches que il ne semble besoin d'en quérir autre fondacion et ass-i treure l'on femmes aroir succédé et paisiblement joy tonte leur vie esdictes seignearies comme la duchesse Jehanne ile Brabant. fille du duc Jehan de Brabant, l'espace de plus de cinquante ans, et combien qu'elle fust mariée, se se faisoient néantmoins par tout son temps toutes lettres touchans les faiz du paîs par son mary le duc Wencelaus comme par son mainbour et ou nom d'elle comme dame et princesse héritière; pareillement succéda es comtez de Haynnau, Hoilande, Zellande etc. ${ }^{a}$ la comtesse Marguerite, femme de Loys, empereur, IIII ${ }^{\ominus}$ de ce nom, et si succéda es dictes terrez et comtez par le trespas du conte Willem, son frère, dont elle fut recene à féaulté et hommage et obtint lettres de l'Empereur sur sadicte investiture par lesquelles fut déclairié qu' elle estoit bien et deuement succédée esdictes seigneuries comme le plus prouchain hoir dudit comte Willem, son frère. Pareillement le duc Anthoinne, due de Brabant etc. ${ }^{a}$, obtint lettres de Wencelaus, roi des Rommains par lesquelles est déclairié qu'il estoit bien deuement et légitimement succédé oudit duchié de Brabant par moïen de femme, assavoir de ladicte duchesse Jehanne et de dame Marguerite, sa mère, nièce de ladicte duchesse Jehanne. et comme dit est dessus jamais ne sera trouvé par cronique, ne autrement que de la succession desdictes terres et seigneuries femme ait este reboutée quant le droit par proucbainneté en est arenu sur femme, ne que à cause de faulte de hoir masle, droit de devolucion ait eu lieu.

Item, et pour monstrer la généalogie ${ }^{1}$ ) de mondit seigneur pour voir l'ordre de sa succession esdictes seigneuries, dira ledit maistre Anthoinne

1) Brabant

Johann III. +1355

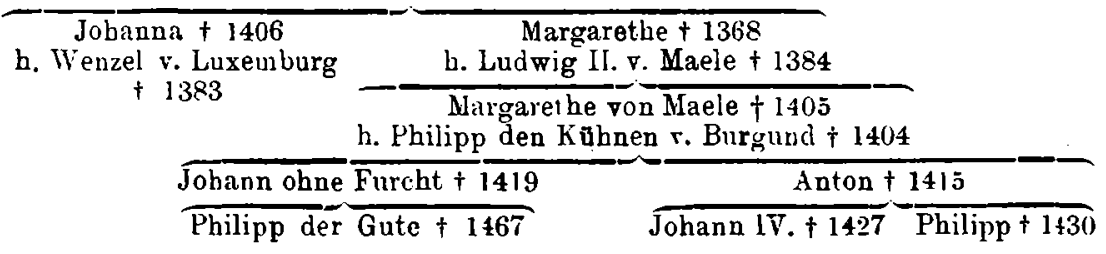


premièrement en tant que touche Brabant etc. ${ }^{3}$ que le duc Jehan de Brahant penultime de ce nom, laissa it son trespas une fille nommée Jehanne qui lui succéda oudit duchir et fut mariee au duc Wencelaus de Behaingne, mais elle n'eust nul enfans et une autre nommee Sarguerite qui fut mariee au comte Loys de Flandres, laquelle ent dudit comte Loys de Flandres une tille nommée Jarguerite qui fut mariée au duc Philippe de Bourgongne, grant père de ınondit seigneur et duquel mariage issy le duc Jehan, ainsné filz dudit duc Philippe de Bourgongne et père de mondit seigneur, et ainsi \& ladicte duchesse Jehanne devoit succeder oudit duchié de Brabant icellui duc Jehan, mais son frère mainsné assavoir le duc Anthoinne y succéda, du consentement dudit duc Jeban de Bourgogne, son frère ainsné, et depuis succédèrent audit dac Anthoinne deux ses enfans, assaroir le duc Jehan ainsné et le duc Philippe mainsné, que tous deux sont trespassez sans boir de leur corps, et audit duc Philippe de Brabant derrenier est succédé mondit seigneur comme son plus prouchain hoir, car ilz estoient filz de deux frères et n'y aroit autre plus prouchain de lui qui venoit et descendoit lu frère ainsné comme dit est.

Item, en tant que touche Haynnau, Hollande ${ }^{1}$ ) et Zellande, est vray que de dame Marguerite, contesse et dame desdits pays et empereys, femme dudit Loys, empereur des Rommains JIII ${ }^{\theta}$ de ce nom, laquelie fut receue à foy et hommage d'icelles terres et seigneuries, et lui succéda le duc Albert son filz, auquel succéda le duc Guillaume, son filz, lequel duc Guillaume avoit une seur nommée Marguerite qui fut mariée au dur Jehan de Bourgoingne, père de mondit seigneur et duquel mariage issy mondit seigneur qui est à présent. Item, aroit icellui duc Guillaume une fille nommée dame Jaques de Bavière qui succéda à sondit père et trespassa sans laissier hoir de son corps: si lai succéda mondit seigneur comme son plus prouchain hoir, au moins comme le plus ainsné de ceulx qui lui estoient en parail degré. Et ainsi appert la généalogie de mondit seigneur pour succéder aux dictes terres et seigneuries comme le plus prouchain en sang et le plus ainsné de ceulx qui estoient en parail degré.

Item, et $s^{\prime} i l$ ad̃enoit que le duc Albert d'Ostriche ${ }^{2}$ ) fust devers l'Empereur à l'advenne dadit maistre Anthoinne, icellui maistre Anthoinne

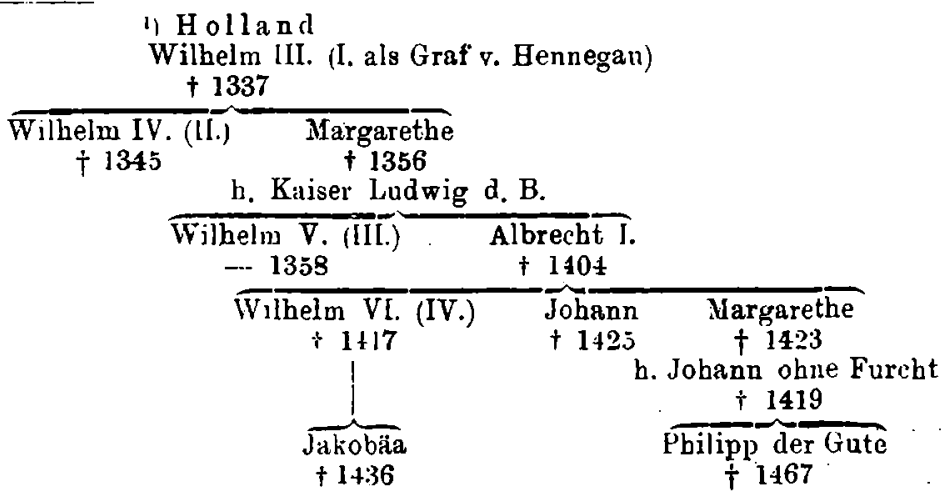

2) Der Bruder Kaiser F'riedrichs, mit dem Philipp im Jahre 1447 einen Vert:ag geschlossen hattte; vgl. Chmel, Gesch. Kaiser Friedrichs Il 473: Chmel, katerialien I $2+7 \mathrm{ff}$. 
se traira devers lui si tost qu'il aura esté la première fois dever's l' Fumpereur. lui baillera lettres de par mondit seigneur contenans créance sur lui et pour sa créance exposer contendera de parler à lui à part ou a.u moins bien secrètement, et lors dira comment a son partement le pardeça estoit venu levers mondit seigneur l'un de ses gens nommé Hughe de Palberch qui avoit apporté a mondit seigneur lettres de créance et aussi copie de trois lettres ${ }^{1}$, assavoir l'une du don que l'empereur lui a fait du droit desdits duchiez, comtez, terres et seigneuries tenues de l'Empire et comme dévolues au droit impérial, l'autre par laquelie l'Empereur lui donne povoir et licence pour icelles terres et seigneuries transporter it cui que bon lui semblera, et la tierce par laquelle l'Empereur lui donne povoir. licence et faculté de appointtier et accorder sur le fait des investitures et hommages desdits duchiez. comtez, terres et seigneuries et surquoy lui avoit esté respondu de par mondit seigneur qu'il mercioit ledit duc Albert et autres thuses que ledit maistre Anthoinne scet et qu'il saura bien dire, et cuidoit bien mondit seigneur besongnier avec ses gens et cunseillers, mais puis que la chose estoit en telx termes qu'il estoit devers l'Empereur, se son plaisir estoit d'entendre aux matières pardeli et qu'il $y$ voulsist besongnier, ledit maistre Anthoinne se offrera prest à ce faire, en délaiant tousjours et reculant par la meilleur manière qu'il pourra tant qu'il ait nourelles de pardeça.

Item, et ou cas que ledit maistre Anthoinne trouvast qu'il ne peust raisonnablement besongner pardelà, il trouvera manière de mettre avant par manière de son propre adviz et sans faire semblant d'en faire charge, la manière d'aliance que l'on scet. et par cc moïen telement faire que autre journée fust prinse pardeça où les gens de l'Empereur et se besoing est du duc Albert, venissent chargiez de tout, et se l'on puet autrement besongnier ès matières dessusdictes, il ne sera besoing d'en parler si non assez légièrement et de soy mesmes pour seulement sentir quelle disposicion en est pardeli ou cas que cy après l'on $y$ voulsist contendre, sans toutevoies emprandre journée à ceste cause.

Item, et quant ledit maistre Anthoinne aura besongnié devers l' Empereur ès matières dessus dictes, il s'en ira devers nostre Saint Père le Pape et lui fera rapport de tout ce qu'il aura besongnié, tant du fait du secours et ayde contre le Turc, comme des autres poins et articles, en merciant nostre dit Saint Père de la grant et singulière faveur qu'il a démonstré à mondit seigneur le duc en la conduitte des choses dessus dictes, en recommandant son fait, dont ledil maistre Anthoinne s'est bien apparceu en pluseurs manières.

Item, contendera icellui maistre Anthoinne de savoir de nostre dit Saint Père quelle sera son entencion de faire et besongnier touchant la dicte matière contre le Turc, et aussi touchant les appaisemens de questions et différens de France et d'autres de pardeçà, affin que icellui maistre Anthoinne en sache faire bon rapport à mondit seigneur.

Item, et s'il entendoit que nostre dit Saint Père fust d'entencion de cueillir et lever ès païs de mondit seigneur les dixiesmes sur les gen;

1) Vgl. die Lirkunden König Friedrichs vom 3., 3. (oder 6.) u. 7. April 1446; Chmel, Reg. Friderici nr. 2058 (Anbang S. LXXXXIV nr. 66); 2059; 2066 (Chmel, Materialien I 203 t.; nr. 77); s. auch Chmel, Gesch. Kaiser Friedrichs II 371), 
d'eglise et les $X X X^{\text {mes }}$ sur les gens laiz, pour les deniers qui en istroient emporter hors desdits païs de monseigneur et applicquer ou bon sembleroit à nostre dit Saint-Père: dira ledit maistre Antboinne que les païs de mondit seigneur le duc sont païs plains de gens populaires fort privilégiez de diverses natures et condicions et si sont païs marchissans a pluseurs autres estranges et divers praïs, régions et contrées et fait on grand doubte que se le fait desdits dixiesmes et $\mathrm{XXX} \mathrm{X}^{\text {mes }}$ estoit mis en termes de par mondit seigneur en sesdits païs, terres et seigneuries que l'on n'y trouvast de l'obstacle et de bien grandes difficultez, et attendu que comme l'on dit nostre dit Saint Père doit envö̈er pardeçà en légacion aucuns de messeigneurs les cardinaulx pour l'appaisement des guerres, divisions et différ et d'Angleterre et autres de ces marches de deça les mons: il semble, à correction, qu'il seroit convenable et expédient que la chose touchant lesdits dixiesmes et $X_{X X}{ }^{\text {mes }}$ fussent mis avant par mesdisseigneurs les cardinaulx et qu ilz eussent charge de ce faire de par nostre dit Saint-Père, affin que ceste matière fust conduitte de par sa Saintteté et s'en pourroit la chose trop mieulx porter.

Item, dira ledit maistre Anthoine à monseigneur l'Evesque d'Arraz et a maistre Pierre Bogaert, procureur de mondit seigneur le duc en court de Romme qu'ilz facent et donnent audit maistre Anthoinne tout l'ayde, conseil, confort, faveur et assistence que bonnement pourront, à faire et poursuir les choses dessusdictes et autres qui cy après s'ensuivent:

C'est assavoir qu'il plaise a nostre dit Saint-Père promouvoir mondit seigneur d'Arraz à l'estat et dignité de cardinal ${ }^{1}$ ), ainsi que mondit seigneur par $c_{j}$ devant lui a escript, en remonstrant les raisons qui pévent et doivent mouroir et dont ledit maistre Anthoinne est bien adverti et mesmement ce que mondit seigneur lai a dit de bouche à son partement de devers lui et quant il print congié de lui, et le ce fera aussi icellni maistre Anthoinne diligence devers messeigneurs les cardinaulx, selon qu' il trouvera estre expédient de faire et entre autres choses dira comment mondit seigneur est adverti que nostre dit Saint Père a de nouvel fait et créé quatre ou cinq cardinaulx sans avoir promen mondit seigneur d'Arraz à lailicte dignité, doni mondit seigneur se donne merveilles, attendu ce qu'il aroit escript à ceste fin et mesmement la nécessité que mondit seigneur le duc a pour le bien de lui et de ses besongnes et affaires et aussi pour le bien de ses pais et subgez que ledit monseigneur d'Arraz soit fait et créé cardinal.

Item, pour ce que nostre dit Saint Père, en faveur de Révérend Père en Dieu l'Evesque de Toul²), chief du conseil de mondit seigneur le duc en l'absence de monseigneur le chancelier, a desjà baillié ses bulles pour faire deffense à ceulx de chapitre de Chalon que la vacation de l'éreschié didit Chalon soy offrant, ilz ne procèdent à aucune élection de futur pasteur pour ce aussi que l'on dit que l'évesque ${ }^{3}$ ) dudit Chalon qui est

1) Erst bei seiner zweiten Kardinalsernennung im Dezember 1461 konnte Pias II. die Wabl Jouttiogs im Kollegium durchsetzen; vgl. sein Schreiben an Herzog Philipp bei Pastor II 727 nr. 37 u. II 109.

2) Wilhelm Filastre erbielt aw 4. September 1460 dıs Bistum Tournay.

3) Johaunes Germain + 1461 Febr. 2. 
à présent est indisposé, il plaise à nostre dit Saint Père avoir mémoir dadit Eresque de Toul quant à ce que dit est et si tost que ladicte vacation escherra et vendra à si congnoissance, promouvoir icellui evesque de Toul audit éveschié devant tont autre.

Item, qu'il plaise à nostre dit Saint Père. aroir poul especialment recommandé maistre Anthoinne de Seufchastel, filz de monseigneur de Neufchastel, mareschal de Bourgogne, lequel maistre Anthoinne est prothonotaire du Saint Siège apostolique et lui estre favorable si avant que bonnement faire le pourra ès requestes qu'il fera bailler et présenter à sa Saincteté pour son avancement et sa promotion.

Item, qu'il plaise à nostre dit Saint Père avoir pour espécialment recommandez Ernoul et François de Lalaing. filz. légitimes dudit messire Simon de Lalaing, touchant leur promocion et arancement en l'église.

Item, qu'il plaise à nos.re dit Saint Père, avoir souvenance de la recummandacion autres foiz faicte par mondit seigneur et pirr ses deux derrenières ambassades en faveur dudit maistre Anthoinne et à celle fin qu'il soit pourveu des dignitez ès églises d' Ltrecht et de Deventer réservées it la disposicion de nostre dit Saint Père.

Item, qu'il plaise à nostre dit Siint Père mettre au néant certainne union obtenue par maistre Gervais D . . . . . doïen de l'église suinte Pharaould de Gand ou prejudice de monseigneur de Cambray ${ }^{1}$, et dont ledit maistre Anthoinne est plainement adverti.

Item, qu'il plaise à nostre dit Saint Père ottroïer ses bulles apostoliques pour deffendre au couvent de Saint Pierre lez Chalon, de l'orire Saint Benoist, l'section de leur abbé quant la vacation de l'abhave premiers escherra par le trespas de l'abbé qui est ì présent, et nota que ladicte abhaye est subgette au Saint Siège apostolique sans moïen.

Item, qu'il plaise ì nostre dit Saint-Père, à la requeste de mondit seigneur le duc, avoir pour singulièrement recommandé maistre Pierre Milet, son secrétaire signant sur le fait de ses finances et prévost de l'église Saint-Pierre d'Aire ${ }^{2}$ ) et lui estre favorable si avant que bonnement faire le pourra ès requestes qu'il fera baillier et présenter à sa Saincteté pour avoir revalidacion de ses grices expectatives et nominacions en ce en quoy elles n'ont sorti effect: pour lesquelles avoir et obtenir il a soustenu de granz fraiz, missions et despens, ì quoy, comme il semble, l'on doit avoir regart. Aussi pour avoir des dispensacions et autrement et pour ce que desjà nostre dit Saint Père, oy le rapport et la recommandacion que lui frent lesdits monseigneur d'Arraz, messire Simon de Lalaing et autres estans avec eulx en ambassarle de la personne dudit maistre Pierre, de la charge qu'il a devers mondit seigneur le duc en ses matières secrètes et autrement et de sa continuelle résidence par devers lui, s'est démonstré bien favorable pour icellui maistre Pierre, ledit maistre Anthoinne, en la présence de mondit seigneur d'Arraz, l'en merciera de par mon dit seigneur.

Fait et commandé par mondit seigneur le duc le premier jour de may l'an mil CCCC soirante, en sa ville de Bruselles.

$$
\text { (signé): } \quad \text { Milet. }
$$

1) Johannes VI., vnehelicher Sohn Johannes' ohne Furcht.

:) Aire-sur-la-Lys (Pas de Calais). 